\title{
Triggering on electrons, photons, tau leptons, jets and energy sums with the CMS Level-1 trigger
}

\section{Sandeep Bhowmik*}

National Institute of Chemical Physics and Biophysics, Tallinn, Estonia

E-mail: sandeep.bhowmikecern.ch

The CMS Level-1 trigger architecture underwent a full upgrade in 2016 in order to maintain and improve the trigger performance in the challenging experimental environment of Run II. It uses very high bandwidth processors with complex, programmable algorithms which increase the efficiency of identifying physics objects while reducing rates. It has successfully delivered very high performance at the high luminosity and associated pile-up condition.

\section{Introduction}

The purpose of the CMS detector is to study proton-proton and heavy-ion collisions produced by the LHC, primarily to search for new particles and physics processes. The trigger system is mainly responsible to select the most interesting collisions amongst the millions occurring per second. CMS has implemented a sophisticated two-level trigger architecture to reduce the rate by a factor of nearly $10^{5}$. The first level (L1) trigger operates at hardware level and uses the information of the calorimeters and the muon chambers. The L1 trigger latency is $3.8 \mu \mathrm{s}$. The High-Level Trigger (HLT) runs on a massive computer software farm with more sophisticated algorithms exploiting the full detector information. The calorimeter trigger algorithms and recent performance with the data collected in 2018 are presented here.

\section{Level-1 Calorimeter Trigger Algorithms [1]}

The CMS electromagnetic calorimeter (ECAL) provides a precise measurement of the energies and positions of incident electrons and photons for both triggering and offline analysis purposes. The energy measured by the hadronic calorimeter (HCAL) is used to help identify and isolate electromagnetic signals. ECAL and HCAL energies are combined to reconstruct hadronically decaying tau leptons, particle jets and energy sums.

The algorithms exploit thoroughly the global view of the calorimeters and the full trigger tower (TT) granularity provided by the upgraded trigger system. Lepton signatures are reconstructed with a dynamic clustering approach instead of a sliding window while particle jets are reconstructed with optimum size. The calorimeter trigger needs an estimate of the energy that should be subtracted from the measured energy for each calorimeter object and additionally a means to remove objects which originate from pile-up particles. The improved response results in sharper efficiency curves and cuts on dedicated isolation variables help to control the rate.

ICHEP 2018, XXXIX International Conference on High Energy Physics

4-11 July 2018

Seoul, Korea

\footnotetext{
* Speaker.

${ }^{\dagger}$ on behalf of the CMS collaboration
} 


\subsection{L1 Electron and Photon Triggers}

Clusters are seeded by local maxima of energy above a fixed threshold. The maximum size of the clusters is limited (at most $8 \mathrm{TTs}$ ) in order to minimize the impact of pile-up energy deposits while including most of the electron or photon energy. Background rejection is achieved by introducing a shape veto on a large variety of clusters produced by the dynamic clustering. The sum of the ECAL $E_{T}$ of the seed and clustered towers is taken as the raw $E_{T}$ of the cluster. A calibration derived from $Z \rightarrow e e$ is applied to this raw energy with factors depending on the $\eta$-position of seed tower, shape and cluster $E_{T}$. The $E_{T}$ deposited in a $6 \times 9$ TTs isolation region is computed excluding the footprint of the $e / \gamma$ candidate. The isolation threshold is currently tuned to reach $90 \%$ of trigger efficiency, constant as a function of pile-up and $\eta$.

\subsection{L1 Tau Lepton Trigger}

Although the footprint of the $\tau$ lepton (decaying hadronically) energy deposit is larger than that of an electron, the dynamic clustering developed for $e / \gamma$ is perfectly adapted to reconstruct the individual decay products of $\tau$ and can subsequently be merged to form signature of $\tau$.

\subsection{L1 Jets and Energy Sum Triggers}

The jet reconstruction algorithm is based on a square-jet approach with a $9 \times 9$ TTs sliding window centered on a local maximum. The window size chosen here is coherent with a cone radius of 0.4 for the offline jet reconstruction algorithm. The jet candidate energy is the sum of all $9 \times 9$ TTs energies. A local pile-up correction technique called "chunky donut" is applied. Jet energy calibration is applied as a function of the jet $p_{T}$ and $\eta$.

\section{Result and Conclusion}

The performance of L1 $e / \gamma$, tau leptons, jets and energy sums is shown in figure 1 . The efficiency turn-on plots are very sharp and consistent with the result of last year.
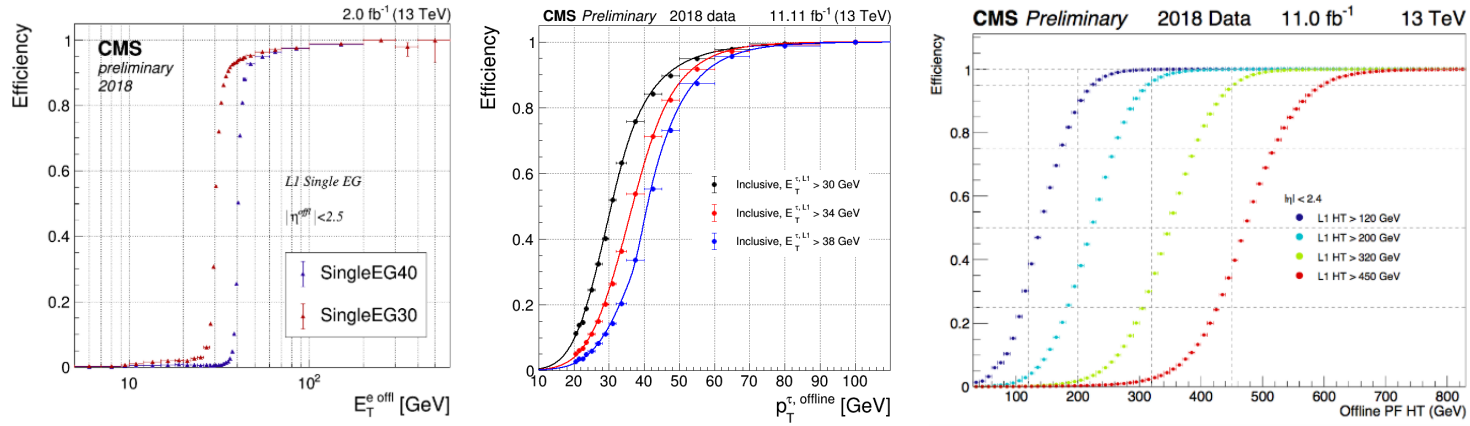

Figure 1: Efficiency turn-on of L1 $e / \gamma$ (left), L1 $\tau$ (middle) and L1 HT (right) w.r.t offline reconstruction

\section{References}

[1] "Triggering on electrons, jets and tau leptons with the CMS upgraded calorimeter trigger for the LHC RUN II", A. Zabi et al., 2016 JINST 11 C02008 